Meta

Journal des traducteurs

Translators' Journal

\title{
Étude comparée des modes de formation des lexies complexes dans deux domaines différents
}

\section{Dorothy Nakos}

Volume 34, numéro 3, septembre 1989

1. Actes du Colloque Les terminologies spécialisées : Approches quantitative et logico-sémantique et 2 . Actes du Colloque Terminologie et Industries de la langue

URI : https://id.erudit.org/iderudit/003687ar

DOI : https://doi.org/10.7202/003687ar

Aller au sommaire du numéro

Éditeur(s)

Les Presses de l'Université de Montréal

ISSN

0026-0452 (imprimé)

1492-1421 (numérique)

Découvrir la revue

Citer cet article

Nakos, D. (1989). Étude comparée des modes de formation des lexies complexes dans deux domaines différents. Meta, 34(3), 352-359.

https://doi.org/10.7202/003687ar d'utilisation que vous pouvez consulter en ligne. 


\section{ÉTUDE COMPARÉE DES MODES DE FORMATION DES LEXIES COMPLEXES DANS DEUX DOMAINES DIFFÉRENTS}

DOROTHY NAKOS

Université Laval, Québec, Canada

\section{INTRODUCTION}

Les vocabulaires spécialisés comportent un nombre élevé de lexies complexes, c'est-à-dire des groupes de mots syntaxiquement liés, séparés par des blancs typographiques, n'ayant qu'un seul sens à la fois dans un contexte donné. Il s'agit normalement d'expressions figées où l'ordre des éléments ne peut pas être inverti, par exemple traitement de données et stimulateur cardiaque. Il existe bien d'autres façons de nommer ce phénomène linguistique: groupe lexical, synapsie, unité de signification, etc. Les terminologues, pour leur part, utilisent de plus en plus l'expression «syntagme» ou «syntagme terminologique» et ils distinguent le «terme», qui est un mot spécialisé, du «syntagme», unité terminologique plus ou moins complexe pouvant, dans de nombreux cas, se décomposer en plusieurs sous-unités. Par exemple, stimulateur cardiaque implanté par voie endocavitaire est formé de deux unités principales: stimulateur cardiaque et voie endocavitaire.

Nous avons analysé les modes de formation de lexies complexes dans deux domaines différents : un domaine connu depuis fort longtemps, la médecine, et un domaine d'introduction récente, l'informatique. Nous présenterons ici quelques particularités de chaque domaine en mettant l'accent sur les analogies et surtout sur les différences existant enre les deux. Nous aborderons uniquement les aspects suivants : 1 . juxtaposition et coordination, 2 . onomastisme et 3 . réduction.

\section{JUXTAPOSITION ET COORDINATION}

Dans les deux domaines à l'étude, nous relevons le modèle classique de juxtaposition d'un adjectif à un substantif, par exemple, terminal portatif, traitement autonome en informatique, médicament hypotenseur, carence digestive, artère nourricière en médecine. Il n'existe pas de grande différence dans ce cas, si ce n'est le fait qu'en médecine, comme nous le savons déjà, le recours aux formes gréco-latines demeure prépondérant et donc les formations savantes comme hystérectomie abdominale, mutisme akinétique, paraplégie spasmodique restent, et resteront, en usage.

Nous n'avons pas relevé, en informatique, de formations aussi savantes, employées régulièrement, pour la simple raison qu'il s'agit d'une discipline nouvelle ayant rapidement progressé, en grande partie grâce aux apports scientifiques et techniques qui ont donné lieu à de très nombreuses publications de langue anglaise, utilisant un niveau de langue usuel et s'adressant à une clientèle diversifiée. Quant à la médecine, la tradition a consacré un niveau de langue savant où les formes gréco-latines ont servi de dénominateur commun à plusieurs langues, y compris l'anglais. Mentionnons que bon nombre d'auteurs ont étudié en détail l'évolution du langage médical et expliqué le rôle des racines et affixes grecs et latins, par exemple Hamburger, Ghazi, Dirckx. 
Notons également le recours à l'image très évocatrice d'un symptôme et de sa cause (étiologie) en médecine, par exemple, collier de Vénus pour désigner la syphillis secondaire, ventre de batracien désignant la cirrhose avancée, ventre de bois, la péritonite, préfet de l'aine, un ganglion de l'aine plus grand que les autres (adénopathie). L'image existe aussi en informatique, mais elle est utilisée de façon moins systématique.

En outre, si l'informatique comporte de nombreux cas de synonymie dus essentiellement au flottement dans l'usage (le vocabulaire n'est pas bien ancré; en outre, l'usage peut changer d'un pays à l'autre), à la simplification de lexies complexes longues (nous y reviendrons plus loin) ou à l'influence d'une autre langue qui peut donner lieu à deux ou plusieurs formes dont l'une dérive de l'anglais, par exemple les synonymes enregistrement sur un support intermédiaire et simplex empruntés à l'anglais, la médecine présente comparativement un nombre bien plus impressionnant de lexies complexes synonymiques (environ $40 \%$ d'après nos calculs, faits à partir des ouvrages de références consultés), dus aux facteurs déjà énoncés auxquels s'ajoutent d'autres facteurs : a) présence de formes savantes ayant la même signification (cas de racines et d'affixes grecs et latins ayant la même valeur, par exemple, abcès infralingual et abcès sublingual); b) existence de niveaux de langue différents, par exemple épithélioma cutanée ou carcinome cutanée d'une part, et cancer de la peau d'autre part; c) importance de lexies complexes onomastiques ou syntagmes onomastiques, c'est-à-dire des groupements lexicaux comportant plus ou moins un nom propre, par exemple maladie de Basedow, typhus de Sao Paulo, fièvre maculeuse brésilienne (nous aborderons ce sujet plus loin; d) présence d'un syntagme général et d'un syntagme spécifique, ce dernier découlant de la découverte des causes des maladies et des syndromes. Par exemple, le syntagme général, syndrome de Banti englobait toute une série d'affections de la rate présentant des traits communs. Au fur et à mesure que les causes des affections, parasites par exemple, étaient connues, le syndrome de Banti s'est précisé et il est devenu fibroadénie de la rate.

Le modèle classique de juxtaposition substantif + un ou plusieurs adjectifs (par exemple, collique néphrétique) côtoie, dans les domaines à l'étude, le modèle de coordination de base où un joncteur sert de lien entre deux termes (par exemple, pince à hémostase), mais il est à noter que le modèle substantif + préposition à + verbe à l'infinitif souvent relevé dans les domaines techniques (par exemple, lampe à souder, machine à écrire) se retrouve très exceptionnellement, notamment en informatique, où le substantif est préféré au verbe, par exemple aiguille d'impression, programme d'analyse, programme de dépistage plutôt que aiguille à imprimer, programme à analyser, programme à dépister. Évidemment, le recours au substantif permet ici de lever l'ambiguïté que le verbe pourrait créer. Nous devons d'ailleurs signaler que d'une façon générale, la tendance est de remplacer de plus en plus le modèle substantif + à + à l'infinitif par un terme simple suffixé (par exemple, déchiqueteuse au lieu de machine à déchiqueter) ou encore par le modèle substantif + à + substantif que nous venons de traiter (par exemple, pistolet à peinture).

Toutefois, nous remarquons une différence essentielle entre les deux domaines à l'étude quant à l'absence de joncteur entre deux substantifs. Ce procédé qui n'a presque pas été relevé dans notre corpus de médecine* se retrouve très souvent en informatique, par exemple, language origine ou langage source, mode système, mode message, liaison quatre-fils, carte détail, fichier bibliothèque, clé protection. L'accolement ne permet pas d'établir avec certitude le type de lien existant entre les deux substantifs. Évidemment, nous pourrions dire que nous sommes en présence d'une formation s'apparentant à la composition, qui existe depuis longtemps en français (par exemple abat-jour) et que le trait d'union entre les deux substantifs finira par s'insérer définitivement dans cet ensemble. Or en informatique, à l'existence des deux substantifs sans joncteur s'ajoute 
souvent celle d'un syntagme formé des mêmes substantifs liés par une préposition (par exemple, langage origine et langage d'origine). Il semble donc dans ce cas que l'absence de préposition est due à une certaine simplification du syntagme où figure la coordination. Il est certain que ce phénomène se retrouve dans d'autres secteurs (par exemple, assurance-vie, maintenant ancré dans la langue).

Rappelons également que l'accolement de substantifs est une ressource particulière à l'anglais; elle engendre d'ailleurs de la confusion dans cette langue comme le souligne Dirckx (1976: 137):

«[...] the English custom of using a noun as an adjective without somehow signalling the hearer or reader that there has been a change in the role of the word gives rise to an incalculable amount of confusion.»

Le langage médical ne peut pas se permettre cette absence de précision et il explicite de façon presque systématique les liens entre les termes (par exemple, facteur d'inhibition de la migration des macrophages). Évidemment, 1'informatique a recours le plus souvent au modèle de coordination traditionnel et s'en sert, tout comme la médecine, pour créer des séries. Nous avons relevé, par exemple, cent un types de programme en informatique: programme d'assemblage, programme de commande, programme de gestion, etc, deux cents types de corps en médecine: corps en demi-lune, corps de Hensen, corps de Lafora, etc.

De plus, la complexification du syntagme permet de hiérarchiser des notions. Par exemple, caractère alphanumérique se divise en jeu de caractères alphanumériques, à son tour divisé en jeu partiel de caractères alphanumériques. Une option peut également être ajoutée, par exemple caractère alphanumérique avec édition, etc. La complexification permet, en outre, de montrer les étapes d'une découverte médicale comme le signale Ghazi 1985: 178):

«[...] le progrès de la néphrologie a permis de distinguer d'abord deux variétés de néphrites, la néphrite aiguë et la néphrite chronique; plus tard la néphrite chronique atrophique; puis la néphrite chronique atrophique de l'enfance. À chaque étape de la découverte médicale, une lexie vient s'ajouter à la lexie existante pour accentuer sa spécificité.»

On peut même ajouter à cette liste glomérulonéphrite et tubulonéphrite qui servent maintenant de base à la classification des néphrites aiguës ou chroniques. Évidemment, les risques de polysémie sont moindres lorsqu'on est en présence de lexies complexes, et surtout de lexies complexes longues. N'oublions pas que le syntagme ou lexie complexe est au point de départ descriptif ou explicatif. La création, par exemple, de deux syntagmes descriptifs comme 1. formation de faisceaux et 2. contraction musculaire involontaire permettrait d'éviter l'ambiguïté du mot polysémique fasciculation qui a justement ces deux valeurs; en fait, une définition aiderait à comprendre qu'il s'agit dans le premier cas de faisceaux de muscles ou de nerfs, dans le second d'une contraction de faisceaux de fibres musculaires... Le syntagme ne peut évidemment pas tout expliciter et, comme le terme, $\mathrm{il}$ choisit un aspect de la réalité à décrire.

Une autre particularité du langage informatique est le recours au syntagme que nous appelons «syntagme en tandem», c'est-à-dire un syntagme composé d'au moins deux éléments d'importance égale. Il existe, dans ce cas, un cheminement entre les deux éléments, par exemple stockage et retransmission, recherche et remplacement, chargement et exécution. Nous incluons également dans cette catégorie les expressions comme retrait-rappel, entrée-sortie, car, dans ces derniers cas, le tiret sert à joindre les deux éléments tout comme le fait la conjonction et; selon nous, il ne s'agirait pas ici d'une formation par composition traditionnelle. 


\section{ONOMASTISME}

D'une façon générale, nous avons vu jusqu'à présent quel était le rôle de la juxtaposition et de la coordination (ou absence de coordination) entre les éléments d'un syntagme dans le domaine de l'informatique et de la médecine tout en soulevant la question de la synonymie et des niveaux de langue. Il serait bon maintenant de souligner une différence essentielle entre ces deux domaines quant au recours au nom propre. En informatique, les syntagmes onomastiques (composés d'au moins un nom propre, rappelons-le) sont rares. Les quelques syntagmes relevés proviennent généralement des mathématiques (algèbre de Boole, par exemple), de la physique ou autre domaine accidentel (par exemple code Baudot, d'un ingénieur qui a donné son nom au code de transmission utilisé par le télex). Nous avons aussi de rares cas où un prénom ou nom peut servir à désigner une réalité dans ce domaine (par exemple Ida, prénom d'une téléphoniste au Manitoba). En informatique, il n'existe certes pas la même tradition ni la même volonté qu'en médecine de nommer des réalités en utilisant des appellations propres. Si en médecine, nous relevons également des appellations propres provenant d'autres domaines comme les mathématiques et la physique, les noms propres servent à désigner toutes les sortes de réalités bien médicales: syndromes (syndrome de Cushing), signes (signes de Babinski), maladie (maladie de Basedow, mal de Pott), sources de maladies (fièvres de Malte), procédés (procédé d'Outard) instruments (appareil de P. Brocq et R. Dulot), colorants (réactif de Schiff), réalités structurales (appareil de Colgi). En outre, contrairement à ce que prétendent certains auteurs comme Hamburger (1982: 137 et ss), le médecin n'est pas la seule personne à laisser son empreinte ; le patient le fait également par l'intermédiaire d'un groupe de médecins (par exemple, facteur Hageman, facteur Christmas, hémoglobine Auberger; il s'agit ici de noms de patients). De plus, un centre de recherche peut laisser son nom à une réalité médicale (par exemple, hémoglobine Hopkins de l'Université du même nom à Baltimore, hémoglobine Hammersmith, de l'hôpital du même nom à Londres, hémoglobine Saint-Antoine, Saint-Antoine désignant un hôpital de Paris) et même un écrivain peut le faire (par exemple, signe de Musset).

Nous pouvons rapprocher ces derniers cas des toponymes. En médecine, les noms de lieux servent essentiellement à désigner des maladies infectieuses (par exemple, fièvre méditerranéenne) et des colorants (bleu de Prusse, rouge Congo déjà cité). La littérature et la mythologie ont aussi un rôle à jouer (réflexe achilléen, collier de Vénus déjà cité, vert Janus). La mythologie, la légende ou la littérature interviennent en informatique surtout quand des spécialistes nomment des langages ou des systèmes. Il s'agit généralement de syntagmes réduits à quelques lettres qui forment un ensemble acronymique. Prenons par exemple CASSANDRE qui vient de langage pour la conception aidée et la simulation des systèmes logiques, leur analyse, description et réalisation, Didon de diffusion de données; par ailleurs EUCLID, progiciel graphique, évoque le mathématicien grec Euclide. L'application de noms propres, comme nous pouvons le constater, ne se fait pas de la même façon dans les deux domaines à l'étude et l'utilisation n'est pas aussi importante dans les deux cas.

\section{RÉDUCTION}

Il reste que l'informatique et la médecine se rejoignent sur le plan de la réduction de la lexie complexe par divers procédés, notamment la siglaison qui se prononce lettre à lettre (par exemple $D D A$ ) et l'acronymie qui se prononce comme un mot (par exemple Didon déjà cité). Dans les deux cas, le sigle figurant dans les textes français est soit emprunté tel quel de l'anglais (par exemple, $A V$ de assisted ventilation en médecine, $D D A$ déjà cité, de digital differential analyser en informatique), soit traduit ( $E C G$ de électro-encéphalogramme, CAO de conception assistée par ordinateur). En outre, si 
l'acronyme est souvent traduit, surtout lorsque cette réalité devient connue du grand public (par exemple $A I D S$ en anglais et SIDA en français), il présente l'avantage de pouvoir rester le même quelle que soit la langue d'origine (par exemple ELISA de enzymelinked immunosorbent assays, ORFĖVRE de outil de reconnaissance des formes et d'expertise sur les vertèbres par le radiologiste en médecine et CASSANDRE, EUCLID, déjà cités, en informatique). Dans ce cas, nous avons des acronymes uniques facilitant la communication entre les scientifiques de divers pays. La seule différence notable entre les deux domaines touche le téléscopage, fréquent en informatique et quasi inexistant en médecine, d'après les ouvrages consultés. Autrement dit, l'informatique a recours très facilement à des expressions comme bit (de binary digit), codet (de coded element), infotecture (de information et architecture) pixel (de picture element), codec (de codeur-décodeur), modem (de modulateur-démodulateur). La médecine préfère expliciter en ayant recours à des formations savantes et des lexies complexes longues, ou encore en utilisant des noms propres, l'ajout de qualificatifs onomastiques permettant alors d'éviter de citer une lexie complexe au long.

Par ailleurs, si en médecine, le syntagme onomastique peut être réduit au nom propre qui agit comme un nom commun, (par exemple, un farabeuf de écarteur Farabeuf et, surtout dans la langue orale, un parkinson de maladie de Parkinson, un golgi de appareil de Golgi, un babinski de signe de Babinski) - et ainsi toute confusion possible quant au sens du terme est évitée grâce à la spécificité du nom propre. En informatique, le syntagme est plutôt réduit au noyau qui, hors contexte, peut avoir une valeur polysémique. Par exemple, bloc de contrôle et même à bloc. De plus, nous avons relevé des cas d'homonymie siglique surtout en informatique (presque pas en médecine). Il est bon de signaler en terminant cette rubrique que les sigles sont systématiquement expliqués dans les articles médicaux consultés, ce qui n'est pas nécessairement le cas en informatique.

\section{CONCLUSION}

En conclusion, nous pouvons dire qu'il existe une grande variété dans les modes de formation des lexies complexes en médecine et en informatique. Toutefois, dans le premier cas, cette variété est due, comme nous l'avons vu, à la longue évolution du langage médical et à la présence de niveaux de langues différentes; dans le second, la variété est le fait de l'absence d'uniformité du vocabulaire. Nous avons vu par exemple, qu'une forme sans joncteur pouvait côtoyer une forme avec joncteur (langage origine, langage d'origine, module d'origine).

En outre, nous pouvons ajouter que la lexie complexe longue cache de fait l'absence du mot. La réduction nous paraît inévitable dans le domaine de l'informatique, accessible à un grand nombre d'usagers; elle l'est moins dans le cas d'un domaine peu accessible comme celui de la médecine où la précision est primordiale, ne serait-ce que pour décrire une maladie. Le recours à des procédés comme l'onomastisme, le lexicalisme complexe savant, permet alors d'obtenir cette précision. 
TABLEAU RÉCAPITULATIF

Ressources de la langue

en informatique en médecine

a) juxtaposition adjectif + substantif

- cas de la synonymie

$\mathrm{X}$

$\mathrm{X}$

oui

- flottement dans

l'usage

- influence d'une autre langue

b) coordination substantif
à + substantif
- accolement
- syntagme «en tandem»
- complexification

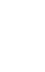

$\mathrm{X}$

X

$\mathrm{X}$

- création de séries

- hiérarchisation

des notions

c) recours au nom propre

$\mathrm{X}$

provenant surtout de disciplines connexes anthroponymes: scientifiques, autres (rare)
- flottement dans l'usage

— influence d'une autre langue

- niveaux de langue différents

- affixes et racines grecs et latins ayant la même valeur

- importance des noms propres

- existence d'un syntagme général puis d'un syntagme spécifique

$$
\begin{gathered}
\text { X } \\
\text { rarement } \\
\text { - création de séries } \\
\text { - hiérarchisation } \\
\text { des notions } \\
\text { - résultat des } \\
\text { découvertes } \\
\text { médicales }
\end{gathered}
$$

$\mathrm{X}$

provenant parfois de disciplines connexes mais désignant essentiellement des réalités médicales anthroponymes: médecins scientifiques patients groupes de recherche autres toponymes:

pays

hôpitaux groupes de recherche autres 
Ressource de la langue

en informatique

en médecine

d) réduction

- siglaison

- acronymie

- téléscopage

- réduction au terme-noyau commun, polysémique

$\mathrm{X}$

- réduction au terme propre spécifique

NOTE

* Les colorants utilisés en histochimic, par exemple rouge Congo font exception ici. Il s'agit dans ce cas de l'accolement d'un nom propre à un substantif commun.

\section{BIBLIOGRAPHIE SOMMAIRE}

\section{Théorie}

DIRCKX, John H. (1976): The language of Medecine. Its Evolution, Structure and Dynamics, New York, Harper \& Row.

GHAZI, Joseph,(1985): Vocabulaire du discours médical, Structure, fonctionnement, apprentissage, Didier Erudition, coll. linguistique, Paris.

HAMBURGER, Jean (1982): Introduction au langage de la médecine, coll. Médecine-Sciences, Flammarion, Paris.

GUILBERT, Louis (1975): La créativité lexicale, coll. Langue et Langage, Larousse, Paris.

GUILBERT, Louis (1965) : La formation du vocabulaire de l'aviation, Larousse, Paris.

KOCOUREK, Rostislav (1982) : La langue française de la technique et de la science, Oscar Brandstetter, Wiesbaden.

«La fertilisation terminologique dans les langues romanes (1987) : actes du colloque de Paris d'octobre 1986», META, Presses de l'Université de Montréal, vol. 32-3, Montréal.

NAKOS, Dorothy (1986): «Quelques aspects de la langue scientifique et technique en anglais et en français», Université Laval, GIRSTERM, Québec.

SAGER, Juan, David DUNGWORTH, Peter McDONALD (1970) : English Special Languages, Principles and Practice in Science and Technology, Oscar Brandstetter, Wiesbaden.

«Table ronde sur les problèmes du découpage du terme (1978): Ve Congrès de l'Association internationale de linguistique appliquée de 1978», Office de la langue française, Éditeur officiel du Québec, Montréal.

«Traduction et terminologie médicales. Medical Translation and Terminology» (1986): META, Presses de l'Université de Montréal, vol. 31-1, Montréal.

VIGER, Gérard, Alix MARTIN (1976) : Le français technique, Librairie Hachette et Larousse, Paris:

\section{Pratique}

a) informatique

AFNOR-APTERM (1980): Traitement de l'information. Vocabulaire complémentaire expérimental, Norme française Z61-101, Paris.

Commission ministérielle de terminologie de l'informatique, Terminologie de l'informatique (1987), AFNOR, Paris.

DE VILLIERS, Marie-Eva (1986) : Vocabulaire du micro-ordinateur, les publications du Québec, Québec.

LETHUILLIER, Jacques (1982) : Informatique, coll. Initiation aux langues de spécialité ; Sodilis, Montréal. MORVAN, Pierre (1986) : Dictionnaire de l'informatique, Larousse, Paris.

Office de la langue française (1983) : Terminologie de l'informatique, Gouvernement du Québec, Québec.

Secrétariat d'État (1982) : Glossary Electronic Data Processing : Lexique informatique, Bureau des traductions, Ottawa.

b) médecine

AMIEL, M., D. DUCASSOU, G. FRIJA, Ph. GRENIER (réd.), (1987): Recherche en imagerie médicale Medical Imaging Research, Ed. INSERM, vol. 160, Paris.

CAMBIER, J., M. MASSON, H. DEHEN (1985) : Neurologie, Masson, Paris, $5^{\mathrm{e}}$ éd. 
GARNIER-DELAMARE (1985): Dictionnaire des termes techniques de médecine, Edisem, St-Hyacinthe, $21^{\mathrm{e}}$ éd.

GLADSTONE, W.J., (1984) : Dictionnaire anglais-français des sciences médicales et paramédicales. EnglishFrench Dictionary of Medical and Paramedical Sciences, Edisem, St-Hyacinthe, $2^{2}$ éd.

MILLER, B.F., C. BRACKMAN KEANE (1987): Encyclopedia and Dictionary of Medecine, Nursing and Allied Health, W.B. Saunders, Toronto.

TAILLEFER, Raymond (1987): «Applications cliniques de la scintigraphie au 131 - MIBG»; l'Actualité médicale, Presse Médic, 3 février 1987, Montréal. 